\title{
Review of: "Smoking cessation reduces systemic inflammation and circulating endothelin-1"
}

\author{
Theodoros Kelesidis ${ }^{1}$ \\ 1 University of California, Los Angeles
}

Potential competing interests: The author(s) declared that no potential competing interests exist.

This is a relatively small study in a single center in US which investigated whether a 12-week smoking cessation intervention would contribute to a long-term reduction in circulating endothelin-1 (ET-1), tumor necrosis factor-alpha (TNF- $\alpha$ ), and interleukin-6 (IL-6) that are instigators of elevated risk of cardiovascular disease (CVD). The investigators compared levels of inflammatory cytokines, ET-1 and cotinine in blood of study participants at baseline, after the 12-week cessation program (end of treatment, EOT), and 12months (12M) following the cessation program. The main findings were that compared to baseline, both plasma TNF- $\alpha$ and ET-1 were significantly reduced at EOT and 12 months following the smoking cessation program. The reduction in serum cotinine was associated with the reduction in circulating levels of ET-1. Despite significant limitations in the study design, the investigators conclude that the reduction in ET-1 and inflammation may in part, contribute to the lower risk of CVD that is observed with smoking cessation.

There are a number of concerns with this study which include the following:

1. The scientific premise that justifies the link between smoking and ET-1 is limited and overall unclear. There is very limited observational data to support the premise and these small studies have shown only a transient link with smoking (cited study PMID: 8139472). The preclinical study that is cited is based on a rat model (PMID: 10822051) and the finding that smoking directly increase the expression of the vasoconstricting endothelin-A receptor, was based on supraphysiological levels of injectable nicotine that do not translate to a much lower systemic exposure of inhaled nicotine (and the measured cotinine levels). The cited study that justifies the statement that smoking increases ET-1 (PMID: 25288367) is a general review (not original study) about the role of ET-1 in inflammation and does not discuss any effect of smoking on ET-1 levels.

2. It is unclear how smoking can have a long-term impact on ET-1 levels to alter CVD risk. Similarly, it is claimed that the reduction in circulating ET-1 limits the number of ligands available to bind to the potent ETAR, thereby attenuating acute vasoconstriction and improving vascular tone but all these studies are studies of acute changes and not long-term changes in pathophysiology. This is important because the authors make claims that the reduction in circulating cotinine over time (12 months) was associated with the reduction in plasma ET-1. This finding can just reflect methodological problems of 
the study rather than justify any mechanistic claims that smoking can affect long term ET-1 and CVD risk.

3. There is no adequate scientific justification (preclinical models and clinical studies) why ET-1 may be a better correlate compared to IL-6. This is problematic because the investigators showed association of smoking cessation with ET-1 changes but not with IL-6, an established biomarker of CVD risk. The investigators overinterpret their findings that "IL-6 was reduced following the 12-week smoking cessation program, although it did not reach statistical significance". The change was small ( $<20 \%)$ and not statistically significant. The investigators overinterpret their findings on the significance of ET-1 with regards to long term CVD risk and there is not adequate justification how it compares with regards to IL6 (negative data).

4. Overall changes in all measures are small $(<20 \%)$, within variability of assays, of unclear clinical significance and can be explained by methodological limitations rather than true biological differences in the setting of smoking cessation.

5. The authors overinterpret their data regarding the changes in blood pressure in the study participants. The changes were small and not statistically significant. Thus, the relevant section in the discussion about blood pressure and ET-1 is theoretical and does not reflect true findings from the study.

6. To further justify the scientific premise that smoking cessation is association with changes in vascular tone that can contribute to long term CVD risk, the investigators should have done studies to explore the impact on blood vessels. It is discussed that inclusion of other vascular measures, such as flowmediated dilation, arterial stiffness or microvascular function "were outside the scope of the current investigation". These studies are essential and within the scope of the current investigation to justify the scientific premise as outlined above.

7. The study design did not consider possible concurrent use of electronic cigarrete smoking or vaping that can have a major impact on the measures. There were no serial blood measurements of the cotinine levels and only 2 timepoints may not capture variable exposures within a 12 -week period. This is a major limitation.

8. The study design did not consider the effect of pharmacotherapy (varenicline) or nicotine replacement treatment (NRT) on the endothelium (PMID: 28549278, PMID: 22744869). The observed differences in plasma ET-1 over time could just reflect direct effect of the pharmacotherapy rather than any clinically relevant effect of "smoking cessation". This is a major limitation.

9. The baseline characteristics of study participants are not adequately described. Important covariables such as potentially other comorbidities (it is not clearly mentioned that the study participants were healthy), prediabetes (many were obese), use of recreational drugs that can impact endothelium, use of other medications such as steroids or diet supplements. Thus, the data cannot be clearly interpreted.

10. The study participants had elevated weight and BMI (25-30). Obesity can impact plasma ET-1 levels and changes over time in ET-1 (PMID: 7698512). Thus, the data cannot be generalized to non-obese smokers. 
11. There is no consideration of the long-term impact of cryopreservation on stability of measured proteins (ET-1, IL-6, TNF-a). These proteins are not very stable (for ET-1 see PMID: 16627560) and the instability can be worse in plasma compared to serum (PMID: 26835245). The reduction in plasma levels of proteins over time could just reflect degradation from long term storage over 12 months and not a biological effect of smoking cessation. This is a major limitation.

12. The analytical performance of the Simple Plex technology used to measure plasma ET-1 has not been validated against established immunoassays.

13. There is no consideration of collinearity between plasma measurements that may limit interpretation of data.

14. There is no consideration of adjustment for multiple comparisons and false discovery rate.

15. It would be clearer if the authors had shown the changes over time within the same person for all the measures. The small sample size $(n=30)$ can allow this presentation of data and that way the withinperson changes over time can clearly be shown (and not just in format of table 3 ).

16. It is unclear why Pearson's correlation was used and not Spearman. Were the data normally distributed? Was log transformation attempted? The statistical analysis section is unclear with regards to this point. By failing to address these issues, the authors tend to overstate their findings. 